setzlichen Entschuldigung liegt nicht schon darin, dass die Rechtsordnung wegen der Ausweglosigkeit der Lage keine verbindliche Entscheidung mehr treffen und deshalb nicht mehr als ein gewissenhaftes Handeln verlangen kann. Die Straflosigkeit ergibt sich vielmehr aus dem Zusammentreffen zweier Schuldminderungsgründe ${ }^{146}$ : Der Täter muss unter einer - der Konstellation von $\$ 35 \mathrm{StGB}^{147}$ vergleichbaren - starken motivatorischen Kraft seiner Gewissensentscheidung handeln und - dies ist wesentlich, um ein unkontrollierbares Ausufern dieses strafbefreienden „Notausgangs" zu verhindern - das kleinere Übel wählen, im Lebensnotstand also eine größere Zahl von Menschenleben retten ${ }^{148}$. Im Fall der Ex-post- Triage wird aber das Unrecht von vornherein nicht gemindert (da nur das Leben von P1 gegen dasjenige von P2 ausgetauscht wird), so dass auch das vom Unrechtsquantum abhängige Schuldmaß nicht entsprechend reduziert ist.
146) Sternberg-Lieben, in: Schönke/Schröder, StGB, 30. Aufl. 2019, vor $\$ 32, \mathrm{Rdnr} .116$.

147) Der entschuldigende Notstand ist von vornherein nicht einschlägig, da es an der von ihm geforderten besonderen Nähebeziehung von Täter und Gerettetem fehlt; sie kann nicht aus der ärztlichen Garantenstellung hergeleitet werden, da der Arzt auch gegenüber dem Erstbehandelten in einer entsprechenden Pflichtenstellung steht.

148) Baumann/Weber/Mitsch/Eisele-Eisele, Strafrecht AT, 12. Aufl. 2016), \$18, Rdnr. 49; Hörnle, in: FS f. Herzberg, 2008, S. 555, 572; Sternberg-Lieben, in: Schönke/Schröder, StGB, 30. Aufl. 2019, vor $\$ 32$, Rdnr. 116; Zimmermann, Rettungstötungen, 2009, S. 292 ff.; Rönnau, in: Leipziger Kommentar, StGB, 12. Aufl. 2006, vor \$32, Rdnr. 356; ohnehin gegen Straflosigkeit einer Tötung durch Umverteilung von Rettungschancen Jäger, ZStW 115 (2003), 765, 779; Neumann, in: Nomos Kommentar, StGB, 5. Aufl. 2017, \$35, Rdnr. 62; Roxin, Strafrecht AT I, 4. Aufl. 2006, \$22, Rdnr. 163; and. hingegen Kühl, Strafrecht AT, 12. Aufl. 2017, \$12, Rdnr. 100 (aber auch Rdnr. 106).

\title{
https://doi.org/10.1007/s00350-020-5614-z
}

\section{Pandemie durch SARS-CoV-2/Covid-19}

\author{
Datenbasis verbessern, Prävention gezielt weiterentwickeln, Bürgerrechte wahren
Matthias Schrappe, Hedwig François-Kettner, Franz Knieps, Holger Pfaff, Klaus Püschel und Gerd Glaeske

\begin{abstract}
Abstrakt
Basierend auf einer mehrdimensionalen Analyse der epidemiologischen, präventiven und juristischen Implikationen der Pandemie durch SARS-CoV-2/Covid-19 werden Handlungsempfehlungen entwickelt. Asymptomatische Übertragungen machen repräsentative Stichproben sowie epidemiologische Untersuchungen der herdförmigen Ausbrüche notwendig. Spezifische Präventionsansätze (Schutz der Risikogruppen) müssen allgemeine Maßnahmen (z.B. Kontakteinschränkungen) ergänzen, die Grundsätze der Risikokommunikation sollten beachtet werden. Die Einschränkung der Grundrechte ist zeitlich strikt zu begrenzen und muss auf transparenten und nachvollziehbaren Abwä-
\end{abstract}

Prof. Dr. med. Matthias Schrappe, Universität Köln,

ehem. Stellv. Vorsitzender des Sachverständigenrates Gesundheit, Venloer Str. 30, 50672 Köln, Deutschland

Hedwig François-Kettner, Pflegemanagerin und Beraterin, ehem. Vorsitzende des Aktionsbündnis Patientensicherheit Berlin, Sarntaler Weg 40, 12209 Berlin, Deutschland

Franz Knieps, Jurist und Vorstand eines Krankenkassenverbands Berlin,

Winterfeldtstr. 25, 10781 Berlin, Deutschland

Prof. Dr. phil. Holger Pfaff, Universität Köln,

Zentrum für Versorgungsforschung,

ehem. Vorsitzender des Expertenbeirats des Innovationsfonds,

Eupener Straße 129, 50933 Köln, Deutschland

Prof. Dr. med. K. Püschel,

Universitätsklinikum Hamburg-Eppendorf,

Institut für Rechtsmedizin,

Butenfeld 34, 22529 Hamburg, Deutschland

Prof. Dr. rer.nat. Gerd Glaeske, Universität Bremen, SOCIUM Public Health, ehem. Mitglied im Sachverständigenrat Gesundheit, Mary-Somerville-Straße 3, 28359 Bremen, Deutschland gungsprozessen beruhen, die rechtsstaatlichen Maßstäben genügen.

\section{Einleitung}

SARS-CoV-2/Covid-19 stellt eine typische Infektionskrankheit dar. Die mittlere Inkubationszeit beträgt 5 Tage, es besteht eine ca. 2 Tage währende präsymptomatische Phase mit hoher Infektiosität, der oligo- bzw. asymptomatische Verlauf ist häufig, hohes Alter und bestehende Vorerkrankungen bedingen eine schlechte Prognose und die Letalität liegt insgesamt unter 1\%. Die Tatsache der möglichen Übertragung durch asymptomatisch Infizierte macht die quantitative Erfassung der Infizierten und Erkrankten sehr schwierig. Zahlreiche Infizierte zeigen auch im weiteren Verlauf keine Symptome oder entwickeln nur die Zeichen eines grippalen Infektes, so dass sie anhand des klinischen Verlaufes nicht erkannt (und evtl. getestet) werden können. In der Folge stellt sich die Identifizierung von Übertragungswegen als problematisch heraus, so dass sich Infektionsherde bilden können, die erst relativ spät als solche erkannt werden.

Wie auch bei anderen Epidemien (z.B. Ebola) sind es zu Beginn gerade Einrichtungen der Gesundheitsversorgung, die als Ausbreitungsort in Frage kommen. Die Patienten wenden sich an diese Institutionen und führen dort zu einer hohen Belastung, die meisten Institutionen sind jedoch noch nicht vorbereitet, es fehlen Schutzmaterialien, und durch Infektion (und Ausfall) von Mitarbeitern kommt es zu einer galoppierenden Dysfunktionalität dieser Einrichtungen. Die damit in Zusammenhang stehenden Eindrücke sind für die öffentliche Rezeption der Epidemie von tragender Bedeutung (etwa die TV-Bilder aus Bergamo und anderen Regionen). In der nächsten Phase kommen dann andere Herde hinzu, z. B. Pflegeheime oder kritische Arbeitsumgebungen (z. B. Schlachthöfe). Sehr schnell müssen jedoch nicht nur die einzelnen Einrichtungen, sondern 
es muss das ganze Gesundheitssystem an die Erfordernisse angepasst werden, wodurch deren spezifische Steuerungsdefizite schlaglichtartig zutage treten (z.B. Zugangsprobleme in Markt-gesteuerten Systemen, Koordinationsprobleme in staatlich oder kooperativ gesteuerten Systemen) ${ }^{1}$.

Nach einer initial oft zögerlichen Reaktion fühlt sich die politische Führung vieler Länder zur Bewältigung dieses in Tagen ablaufenden Anpassungsprozesses gezwungen, sehr stark auf exekutive Ausnahmerechte zu setzen, wobei der nationale Geltungsrahmen stark in den Vordergrund geschoben wird. Kompetenzverschiebungen von der Legislative zur Exekutive und Konflikte mit den Grundrechten in den demokratischen Systemen sind weltweit die Folge. Allerdings kennt das Grundgesetz in Deutschland keine speziellen Vorschriften für einen Ausnahmezustand im Falle einer Pandemie. Daher gelten sowohl die staatsorganisationsrechtlichen Bestimmungen als auch die individuellen Grundrechte unverändert weiter, auch wenn selbstverständlich Beschränkungen als Folge von Abwägungsprozessen zulässig sind. Gerade die anfängliche Unsicherheit über die Wirkungen der Pandemie rechtfertigt auch einschneidende Beschränkungen, wenn Grundrechte untereinander kollidieren oder gegen staatliche Schutzpflichten abzuwägen sind. Solche Abwägungsentscheidungen verlangen jedoch eine legitime Rechtfertigung, die Beachtung der Wesensgehaltgarantie, die bestmögliche Transparenz über die zugrunde gelegten Fakten und wissenschaftlichen Erkenntnisse und eine ausreichende parlamentarische Kontrolle unter den Augen der Öffentlichkeit. Je länger Beschränkungen andauern, desto ausgeprägter ist die Verpflichtung zu kontinuierlicher Evaluation, speziell in Bezug auf die Beachtung des Grundsatzes der Verhältnismäßigkeit. Dem tragen auch die grundgesetzlich geschützten Möglichkeiten Rechnung, (einstweiligen) Rechtsschutz und gerichtliche Kontrolle zu erlangen ${ }^{2}$.

Die von den Verfassern dieses Beitrags am 5.4.20203 und 3.5.2020 vorgelegten Thesenpapiere wählen analog zum Verfahren der Technikfolgen-Abschätzung einen mehrdimensionaler Zugang ${ }^{5}$, der neben naturwissenschaftlichen bzw. medizinisch-pflegerischen Aspekten auch Public Health-, sozialwissenschaftlichen oder juristischen Sichtweisen Raum gibt. In diesem Sinne verstehen beide Thesenpapiere die Pandemie nicht allein als biologischmedizinisches, sondern ebenso als gesellschaftlich-soziales Phänomen: in Bergamo konnte man nicht nur eine Viruserkrankung, sondern ebenso die Reaktion einer (lokalen) Gesundheitsversorgung mit ihren gesellschaftspolitischen Rahmenbedingungen beobachten. Sinnvolle Präventionsanstrengungen müssen diese Breite der Problematik berücksichtigen.

\section{Epidemiologie}

Epidemiologische Häufigkeitsmaße, die Eigenschaften der Testverfahren und Kennzahlen für die Dynamik der Epidemieentwicklung bilden die Grundlage für jegliche präventive Strategie. In diesem Zusammenhang muss es als ein schwerwiegendes Versäumnis bezeichnet werden, dass in Deutschland nicht bereits Anfang März mit einer zufallsgesteuerten Kohortenstudie auf nationaler Ebene begonnen wurde, obwohl bereits damals klar war, dass es eine hohe Rate asymptomatischer bzw. oligosymptomatischer Verläufe gibt und man nur durch eine Kohorte in der Lage sein wird, verlässliche Aussagen zur Häufigkeitsentwicklung zu machen. Stattdessen wird bis heute die Häufigkeit der Infektion mit anlassbezogenen Testsystemen beschrieben (Zahl täglicher Neuerkrankungen, kumulative Häufigkeit, kumulative Sterblichkeit etc.), bei denen es nicht möglich ist, den Testumfang als maßgeblichen Einflussfaktor auszuschließen (mehr Teste führen auch bei gleichbleibender Häufigkeit zu einem Anstieg der Zahlen). Dringend $\mathrm{zu}$ fordern wäre daher zumindest die Angabe der täglich durchgeführten Tests. Zusätzlich sorgt die kumulative Darstellung für eine übermäßige Dramatisierung, da auf diese Weise der Eindruck eines unabänderlichen Anstiegs vermittelt wird. Welchen Vorteil eine Kohortenuntersuchung aufweist, wird in der Island-Kohorte deutlich ${ }^{6}$. So konnte man dort genaue Angaben zur Altersabhängigkeit, zur Häufigkeit der Symptome (einschließlich des asymptomatischen oder oligosymptomatischen Verlaufs ähnlich eines grippalen Infektes) und zum Krankheitsverlauf machen, immer bezogen auf die Gesamtheit der untersuchten, zufällig ausgewählten Personen.

Eine wichtige Rolle spielt natürlich die Sterblichkeit, wo die klassische epidemiologische Problematik darin besteht, die sicher oder wahrscheinlich Corona-bedingten Todesfälle von der Sterblichkeit an anderen Erkrankungen bei positivem SARS-CoV-2-Nachweis abzugrenzen (analog zu Zurechenbarkeit z. B. bei Todesfällen im Zusammenhang mit nosokomialen Komplikationen im Krankenhaus ${ }^{7}$ ). Es soll dahingestellt bleiben, warum das RKI initial von einer Obduktion abgeraten hat; im vorliegenden Thesenpapier 2.0 wird jedenfalls eine Systematik für die Darstellung dieses entscheidenden Zusammenhangs vorgeschlagen.

Naturgemäß überschätzen anlassbezogene Stichproben die Häufigkeit und die Krankheitsschwere, da in erster Linie symptomatisch Infizierte (Erkrankte) erfasst werden, während Infizierte ohne Symptome nur in Ausnahmefällen identifiziert werden können. Allerdings kommt der ganz grundsätzliche Gesichtspunkt hinzu, dass derartige Untersuchungen zur Prävalenz immer dazu neigen, die tatsächliche Rate von Neuerkrankungen (sog. Inzidenz) zu überschätzen. Der Grad der Überschätzung wird dabei vor allem durch die Dauer der Erkrankung bedingt: umso länger die Erkrankung dauert, umso höher ist die Chance, durch eine Querschnittsbetrachtung an einem bestimmten Tag das Vorliegen einer Erkrankung festzustellen. Dieser Effekt verschiebt auch das beobachtete Krankheitsspektrum, denn da im Regelfall die Krankheitsschwere mit der Dauer der Erkrankung zunimmt, ist es durch die derzeitigen Verfahren wahrscheinlicher, schwere Verläufe zu erkennen, als Patienten, die z.B. nur wenige Tage Test-positiv sind. Im Zusammenhang hiermit muss die Bedeutung der PCR (das gängige Testverfahren) in der späten Krankheitsphase problematisiert werden, wenn die Symptome bereits abgeklungen sind und eine Immunität durch Antikörper bereits aufgebaut ist. Um die Dauer der Quarantäne und die Zahl der Personen, die einer Quarantäne unterworfen werden, auf das notwendige $\mathrm{Maß}$ zu reduzieren, sollte mit hoher Dringlichkeit untersucht werden, ob in dieser Konstellation noch replizierbare Viren nachweisbar sind (z. B. durch eine positive Viruskultur). Ist dies nicht der Fall, könnte die Quarantänedauer deutlich vermindert werden.

Für die allgemeine Teststrategie sind folglich zwei Ansätze zu unterscheiden, zum einen die o.g. repräsentative Stichprobe (als Standardverfahren), zum anderen die Nachverfolgung der Infektionswege mit dem Ziel, diese möglichst

1) Schrappe/François-Kettner/Gruhl/Knieps/Pfaff/Püschel/Glaeske Thesenpapier 2.0, 3.5.2020, https://www.monitor-versorgungsforschung.de/efirst/schrappe-etal_covid-19-Thesenpapier-2-0.

2) Katzenmeier, MedR 2020, $461 \mathrm{ff}$.

3) Schrappe/Francois-Kettner/Knieps/Pfaff/Püschel/Glaeske: https://www. monitor-versorgungsforschung.de/efirst/schrappe-etal_covid-19Thesenpapier-2-0.

4) Schrappe/Francois-Kettner/Gruhl/Knieps/Pfaff/Glaeske: MonitorVersorgungsforschung, online-first, doi: 10.24945/MVF.3.20.18660533.2224.

5) Gerhardus/Stich, ZEFQ 2008, 77 ff.; Francke/Hart, ZEFQ 2008, $63 \mathrm{ff}$.

6) Gudbjartsson et al., N. Engl. J. Med. https://doi.org/10.1056/NE JMoa2006100.

7) Gastmeier et al., DMW 141, 2016, $421 \mathrm{f}$. 
schnell zu unterbrechen. Bzgl. des zweiten Ansatzes ist derzeit eine Ausweitung der täglich angewandten Tests geplant. Allerdings ist aufgrund des sog. Bayes'schen Theorems gerade bei der z. Zt. zu beobachtenden Abnahme der Infektionshäufigkeit in der Bevölkerung mit einer groBen Zahl falsch-positiver Ergebnisse zu rechnen, soweit die Teste auch nur geringste Spezifitätsproblemen aufweisen. Die Folge wäre die absehbare Überlastung des öffentlichen Gesundheitswesens durch sog. falsch-positive Ergebnisse, d.h. durch ein Ansprechen des Tests, obwohl keine Infektion vorliegt (Beispiel: bei angestrebten 4,5 Mill. Tests pro Woche würden bei einer Spezifität von $95 \%$ und einer Prävalenz von 1:100 pro Woche 222.750 falsch-positive Ergebnisse auftreten). Vorzuziehen ist es daher, die nicht-repräsentative Suchstrategie zur Nachverfolgung von Infektionswegen eng an Symptomatik und Kontakt auszurichten.

Die methodischen Mängel der Häufigkeitsmaße und der Testinstrumente haben eine direkte Auswirkung auf die Kennzahlen, die die Dynamik der Epidemie beschreiben, in erster Linie auf die Reproduktionszahl. Da zur Berechnung dieser Größe zwei Häufigkeitswerte miteinander in Relation gesetzt werden, die im Abstand von einigen Tagen erhoben werden, ergibt sich eine Abhängigkeit von allen Maßnahmen, die speziell den zweiten Wert beeinflussen. Zum Beispiel würde eine Einstellung der Testdurchführung den R-Wert sofort auf null absinken lassen (keine weiteren gemeldeten Fälle), bei einer (derzeit ja beabsichtigten) Ausweitung des Testumfanges aber zwangsläufig zu einer Erhöhung des R-Wertes führen, da eine Ausweitung der Stichprobengröße von einem Anstieg der gemeldeten Fälle gefolgt ist. In diesem Zusammenhang ist die verzögerte Kommunikation des Abfalls des R-Wertes bereits vor dem 23.3.2020 bemerkenswert $^{8}$, vor allem da die gemessenen R-Werte eine Situation zu einem Zeitpunkt vor 14 Tagen widerspiegeln.

Diese allgemeinen Grundsätze lassen sich direkt auf konkrete Fragestellungen anwenden. Die Kenntnis der Rate asymptomatischer Infektionen bzw. der Dunkelziffer ist von enormer Bedeutung, da diese Rate die Infektionslast der Bevölkerung bestimmt und gleichzeitig das Erkennen von Infektionsketten erschwert. Nach der vorliegenden Literatur muss mit einer hohen Rate asymptomatisch Infizierter gerechnet werden, wenn einmalig getestet wird (bis über $50 \%$ ). Ein Teil der Infizierten entwickelt später noch Symptome, die aber u. U. so unspezifisch sind, dass sie nicht zur Diagnose führen; genauere Daten sind für Deutschland noch nicht vorhanden, da Kohortenuntersuchungen fehlen. In Zukunft wird durch spezifische Antikörpertests eine Abschätzung der Zahl von Personen möglich sein, die bereits infiziert waren.

In der gegenwärtigen Diskussion um die Lockerung des Shutdown steht die Infektion mit SARS-CoV-2/Covid-19 im Kindesalter ganz im Mittelpunkt. Es sind hier Studien mit einem Patienten-/Populations-relevanten Endpunkt (sog. Outcome, z.B. Mortalität, Erkrankungsrate) von Studien abzugrenzen, die sog. Surrogatmarker als Endpunkt nutzen (z. B. Viruslast). Diese Unterscheidung, die im Mittelpunkt der Evidenz-basierten Medizin ${ }^{9}$ und der Versorgungsforschung ${ }^{10}$ steht, hat in den letzten Jahrzehnten deutlich zur Verbesserung klinischer Studien und deren Verständnis beigetragen. Zur Covid-19-Infektion im Kindesalter lässt die umfangreiche, Outcome-bezogene Literatur eine relativ sichere Aussage zu: Kinder werden seltener infiziert, sie werden seltener krank, die Letalität liegt nahe bei null, und sie geben die Infektion wahrscheinlich sogar seltener weiter. Wenngleich das klinische Bild von Covid-19 in der medizinischen Betreuung natürlich eng beobachtet werden muss, so steht der Öffnung der Schulen und Betreuungseinrichtungen unter Beachtung entsprechender hygienischer Standards und unter wissenschaftlicher Begleitung nichts im Wege.
Wie eingangs erwähnt, breitet sich die SARS-CoV-2/ Covid-19-Epidemie nicht nur homogen in der Bevölkerung aus, sondern tritt zunehmend in Form einer nosokomialen Ausbreitung in Institutionen (z.B. Gesundheitswesen, Pflege- und Betreuungseinrichtungen) und in Form einer herdförmigen Ausbreitung in sog. Clustern auf. Unter den Patienten/Bewohnern sind zahlreiche Infektionen und eine hohe Mortalität zu beobachten, aber auch das Personal weist eine hohe Krankheitslast auf und berichtet zudem über sehr schwierige Umfeldbedingungen (z.B. fehlendes Schutzmaterial). Dieser herdförmige Ausbreitungstyp ist in seinem Muster nicht vorhersehbar (sog. Emergenz) und muss bei den Präventionsmaßnahmen einen der wichtigsten Schwerpunkte darstellen.

Das gegenwärtige Krankheitsgeschehen der SARSCoV-2/Covid-19-Infektion ist als Problem des gesamten Gesundheitssystems anzusehen. Es sind bereits jetzt Unterschiede in der Bewältigung der Pandemie sichtbar, die durch Erreger- oder Wirtseigenschaften kaum erklärbar erscheinen, sondern die Gegebenheiten der lokalen und nationalen Konfiguration und Funktionsweise des jeweiligen Gesundheitssystems widerspiegeln. Dies gilt nicht nur für die Gegenwart, sondern es kann als ausgemacht gelten, dass die SARS-CoV-2/Covid-19-Epidemie auch die zukünftige Entwicklung der nationalen Gesundheitssysteme prägen wird, wobei die Steigerung der Resilienz (Reaktions- und Widerstandsfähigkeit) des Gesundheitssystems gezielt einbezogen werden muss.

\section{Prävention}

Bereits in Thesenpapier 1.0 v. 5.4.202011 wird die These aufgestellt, dass allgemeine Präventionskonzepte (z.B. Schulschließungen, social distancing etc.) durch Zielgruppenspezifische Konzepte ergänzt und später abgelöst werden müssen. In Version 2.0 des Thesenpapiers ${ }^{12}$ wird daher das Thema der spezifischen Präventionsansätze vertieft und gegenüber den derzeit aktuell diskutierten Konzepten abgegrenzt, die sich wie folgt beschreiben lassen:

- Sog. Herden-Immunität: es wird die Entwicklung einer natürlichen Immunität durch Ausbreitung von Covid-19 beabsichtigt (mit der Gefahr einer unkontrollierten Entwicklung); dieses Konzept wird hier wegen seiner schlechten Steuerung nicht weiter diskutiert.

- Die Strategie der Abflachung (mitigation) durch allgemeine, universell angewandte Präventionsmaßnahmen (social distancing etc.). Diese Maßnahmen weisen das Paradoxon auf, dass sie umso länger andauern müssen, umso erfolgreicher sie sind, da sonst eine ,zweite Welle" befürchtet wird. Wegen dieser Problematik besteht die unausgesprochene Tendenz, die mitigation in eine Strategie der Eradikation (suppression) zu überführen; diese Strategie zeichnet sich in Deutschland derzeit ab. Durch ein vollständiges contact tracing, angekündigt im Beschlussprotokoll der Konferenz der Bundeskanzlerin und der Ministerpräsidenten/innen v. 15.4.2020, soll jede Infektion aufgespürt und jede Infektionskette unterbrochen werden. Es muss jedoch stark bezweifelt werden, dass eine Eradikation in einer hochgradig

8) An der Heiden/Hamouda, Epid. Bull. 17, 2020, 10-15.

9) Fleming, Surrogate Endpoints and FDA's Accelerated Approval Process. Health Aff. 24, 2005, 67-78.

10) Schrappe/Pfaff, in: Pfaff, Nengebauer, Glaeske, Schrappe, Lehrbuch Versorgungsforschung, 2. Aufl. 2017, S. 1-68.

11) Schrappe/Francois-Kettner/Gruhl/Knieps/Pfaff/Glaeske, MonitorVersorgungsforschung, online-first, doi: 10.24945/MVF.3.20.18660533.2224 .

12) Schrappe/Francois-Kettner/Knieps/Pfaff/Püschel/Glaeske, https://www. monitor-versorgungsforschung.de/efirst/schrappe-etal_covid19-Thesenpapier-2-0, s. Kapitel 3.4. 
(auch international) vernetzten Gesellschaft wirklich umsetzbar ist, ohne die Freiheit der Person und die Grundrechte dauerhaft einzuschränken. Man muss hier ganz besonders den Zeithorizont berücksichtigen, denn selbst wenn im kommenden Jahr (2021) eine Impfung zur Verfügung stehen sollte, würde die Impfung der Bevölkerung bei einer geschätzten Zahl von 60 Millionen nicht-immunen Einwohnern bei angenommenen 60.000 Impfungen pro Arbeitstag ca. vier Jahre benötigen (ohne Behandlung von Impfkomplikationen, ohne Mehrfachimpfung). Die Schäden für die Gesellschaft durch einen derartig langen lockdown wären vermutlich irreparabler Natur.

Die Autorengruppe des Thesenpapier 2.0 schlägt daher ein spezifisches Präventionskonzept vor, das als ,,dritter Weg““ auf der Strategie der Abflachung basiert, diese jedoch nicht in eine Eradikation überführt, sondern durch spezifische Präventions- und Schutzmaßnahmen für die Bevölkerungsgruppen (und Institutionen) mit dem größten Risiko ergänzt, die dem Ziel der positiven Unterstützung verpflichtet sind und sich klar von Konzepten der „Ausgrenzung“ etc. absetzen. Eine solche Strategie muss auf der Basis einer modernen Risikokommunikation von der Terminologie der Sanktion und Absonderung zu einer Politik der positiven, materiellen und immateriellen Unterstützung und Autonomiesteigerung übergehen.

Die mitigation-Strategie mit universellen Präventionsmaßnahmen weist jedoch nicht nur Defizite hinsichtlich der fast zwangsläufigen Überführung in ein Eradikationskonzept und der daran zu knüpfenden Fragen zur Machbarkeit massive Defizite auf, sondern es bestehen auch schwerwiegende Zweifel an dessen Wirksamkeit. Methodisch sind positive Auswirkungen des Lockdown auf den Verlauf der Epidemie sehr schwer abzuschätzen, denn einfache unkontrollierte Beobachtungen über den Zeitverlauf reichen nicht aus. Die Inhomogenität des Vorgehens im internationalen Vergleich z.B. zwischen Ländern der EU sollte eigentlich die Beobachtung von Unterschieden zulassen, die auf einzelne Komponenten des Lockdown zurückzuführen wären, dies lässt sich jedoch nicht feststellen. Eine Studie nach dem Design eines historischen Vergleichs in Italien kam zwar zu dem Ergebnis, dass der Lockdown zu einer Verringerung der Rate PCR-positiver Personen von 2,6 auf 1,2\% geführt hat ${ }^{13}$, aber weitere Studien müssen folgen.

Es ist allerdings notwendig, neben der Wirksamkeit auch die zu erwartenden unerwünschten Nebeneffekte mit einzuschlieBen (Effizienz). Hier ist zunächst ein Zurückdrängen bzw. eine weniger effektive Versorgung von nicht durch SARSCoV-2/Covid-19 bedingten Erkrankungen zu beobachten, das durch Umorganisation der Gesundheitseinrichtungen zugunsten der Versorgung von Covid-19-Kranken und zur Vorhaltung von Reservekapazitäten zustande kam (z.B. Rückgang der Schlaganfall-Patienten) und nach ersten Studien zu negativen Effekten bei diesen non-Covid19-Erkrankten führen ${ }^{14}$. Nicht weniger bedeutend sind die psychosozialen Folgen der Präventionsmaßnahmen, vor allem die damit verbundene starke soziale Isolation und die damit einhergehenden Einsamkeitsgefühle ${ }^{15}$ Die Folgen solcher Belastungen können von Beeinträchtigungen der Lebensqualität und des Wohlbefindens über erhöhte Krankheitsanfälligkeit bis hin zu einem erhöhten Mortalitätsrisiko führen ${ }^{16}$. Das Risiko, einen vorzeitigen Tod zu erleiden, ist bei sozial isolierten alten Menschen gegenüber nicht isolierten alten Menschen deutlich erhöht ${ }^{17}$.

Um ihre Wirksamkeit zu steigern, wird wiederholt empfohlen, die universellen Präventionsmaßnahmen durch technische Instrumente $\mathrm{zu}$ ergänzen. In Thesenpapier 1.0 wurde auf die datenschutzrechtlichen und die Privatsphäre betreffenden Implikationen hingewiesen, die gerade bei Daten zum Gesundheitszustand von größter Bedeutung $\operatorname{sind}^{18}$. Zusätzlich muss klargestellt sein, dass diese sog. Co-
rona-Apps auf einem rein Technik-zentrierten Verständnis von Prävention aufsetzen. Ein solches monodimensionales Konzept ist nach den wissenschaftlichen Erkenntnissen der letzten Jahre (s. Improvement Science, Implementierungsforschung ${ }^{19}$ ) jedoch von äußerst beschränkter Wirksamkeit. Stattdessen sind sog. komplexe Mehrfachinterventionen (Complex Multicomponent Interventions, CMCI) zu verwenden, die auf den Prinzipien des sozialen Lernens, der Organisationstheorie und Wahrnehmungspsychologie beruhen ${ }^{20}$ und ganz besonders die Autonomie der Patienten bzw. im Fall Covid-19 der Bevölkerung betonen. Die Erfahrungen auf dem Gebiet Patientensicherheit und infection control haben gezeigt, dass diese Konzepte insbesondere bei solchen Ereignissen wirkungsvoll sind, bei denen - wie im Fall Corona - komplexe Prozesse und emergente, d.h. nicht vorhersehbare und nicht nach einem nachvollziehbaren Muster auftretende Ereignisse im Mittelpunkt stehen.

Vor diesem Hintergrund legt die Autorengruppe in ihrem Thesenpapier 2.0 als „,dritten Weg“ ein spezifisches Präventionskonzept vor, das die initial eingesetzte Abflachungsmaßnahmen rasch durch einen besonderen Schutz von Risikogruppen ergänzt, die nach dem bisherigen Kenntnisstand durch hohes Alter, Komorbidität, nosokomiale Risiken und Kontakt mit Infektionsherden (Clustern) gefährdet sind. Um ein Konzept für solche Schutzvorkehrungen zu entwickeln, ist zunächst ein Modell über die infrage kommenden Einflussfaktoren sowie die zu erwartenden Ergebnisse auf den verschiedenen Ebenen zugrunde zu legen. Die Autorengruppe hat hier Bezug auf das Basismodell der Versorgungsforschung genommen (sog. Throughput-Modell ${ }^{21}$ ). Dort werden Präventionsmaßnahmen (neben Patienten-, Organisations- und Erregereigenschaften) zu den sog. Input-Faktoren gerechnet und stehen im Wechselspiel mit sog. Kontextfaktoren. Das Modell kann hier nicht in seiner Gesamtheit geschildert werden, es sei nur darauf hingewiesen, dass alle Maßnahmen zur Prävention nur auf dem Boden ihres Kontextes wirksam werden können. Präventionsmaßnahmen können noch so gut durchdacht und auch implementiert sein, werden die Risiken z. B. aufgrund fehlender Motivation initial nicht adäquat erkannt oder gar geleugnet, oder ereignen sich Fehler in der Kommunikation, oder ist eine Benennung von Risiken sogar sanktionsbewehrt, dann besteht die Gefahr, dass jede Prävention wirkungslos wird oder gar zur zusätzlichen Gefährdung führt. Hinzu kommt, dass die Entwicklung einer Epidemie durch sog. emergente Ereignisse charakterisiert ist (z.B. das Auftreten von Clustern), die auftreten ohne dass man den Zeitpunkt oder Ort vorher benennen kann (eine Folge der Komplexität). Daher sind paradoxe Effekte an der Tagesordnung; entsprechend wird z.B. diskutiert, dass zu einem frühen Zeitpunkt der Pandemie einige europäische Länder durch Schließung ihrer Flughäfen für Reisende aus China deshalb Infizierte bei der Einreise nicht mehr erkennen konnten, da diese über andere europäische Flughäfen umgebucht hatten, um sich

13) Lavazzo et al., medR xiv. 2020.4.17.20053157.

14) Ding et al., Ped. Blood Cancer 2020, submitted.

15) Röhr/Müller/Jung/Apfelbacher/Seidler/Riedel-Heller, Psychiat. Prax. 47, 2020, 179-189.

16) House/Landis/Umberson, Science 241, 1998, 540-545; Holt-Lunstad/Smith/Baker/Harris/Stephenson, Perspectives on psychological science, 10, 2015, 227-237.

17) Saito/Kondo/Kondo/Ojima/Hirai, Social Sci. Med. 75, 2012, 940945.

18) Schrappe/Francois-Kettner/Gruhl/Knieps/Pfaff/Glaeske, MonitorVersorgungsforschung, online-first, doi: 10.24945/MVF.3.20.18660533.2224.

19) Wensing, ZEFQ 109, 2015, 97-102.

20) Übersicht bei Schrappe, Aktionsbündnis Patientensicherheit e. V. (Hrsg.), APS-Weißbuch Patientensicherheit, 2018, S. $466 \mathrm{ff}$.

21) Schrappe/Pfaff, in: Pfaff/Neugebauer/Glaeske/Schrappe, Lehrbuch Versorgungsforschung, 2. Aufl. 2017, S. 15. 
dieser Regelung zu entziehen. Wirkungsvolle Prävention muss daher den Kontext bedenken und außerdem das Phänomen der Emergenz berücksichtigen; monodimensionale Konzepte sind hier grundsätzlich im Nachteil.

Eine wichtige Option, den Konnex zwischen Prävention und Kontext positiv zu beeinflussen, besteht in der Kommunikation bzgl. der geplanten Maßnahmen. Besonders in Zeiten gesellschaftlicher Verunsicherung sind Sprache und Kommunikation wichtige und verantwortungsvoll $\mathrm{zu}$ benutzende Instrumente zur Steuerung von Verhalten und gesellschaftlichen Prozessen ${ }^{22}$. Im Sinne einer abwägenden Risikokommunikation ist es wenig erfolgversprechend, einen Kommunikationsrahmen (framing) zu wählen, der auf einer dauerhaften, unabänderlichen Bedrohungssituation beruht und mit Instrumenten arbeitet, die in erster Linie auf Sanktionen aufbauen. Vorzuziehen sind positive Botschaften, die auf die Lösungskompetenz der Bürger und Bürgerinnen Bezug nehmen, deren Autonomie betonen und die Würde der Person in den Mittelpunkt stellen.

Die Basis der Entwicklung von spezifischen Präventionsansätzen wird durch die transparente und sinnvolle Definition der Risikogruppen gelegt. Um in die Diskussion einzutreten, legt das Autorenteam einen Entwurf eines Risiko-Scores vor. Mehrdimensionale Scores sind am ehesten in der Lage, unterschiedliche Eigenschaften wie Alter, Komorbidität, nosokomiales Risiko und Cluster-Zugehörigkeit zu kombinieren. Es kann folglich nicht darum gehen, anhand eines einzigen Merkmals (z. B. Alter über 60 Jahre) Bevölkerungsgruppen zu definieren, für die ein hohes Risiko angenommen wird, denn in dieser Gruppe finden sich Personen mit einem gesundheitlich sehr guten Zustand genauso wie schwer kranke Personen, die tatsächlich einer Unterstützung bedürfen. Man sollte daher mit den Gruppen mit dem höchsten Risiko beginnen, z. B. multimorbide Bewohner von Pflegeheimen oder Personen in der ambulanten, häuslichen Pflege. Diese Gruppen können Unterstützung finden, indem man ihnen z.B. Schnelltests für Besuchsregelungen finanziert, Zeitslots für den Besuch von Einrichtungen im öffentlichen Raum reserviert, und ihnen belastbar versichert, dass sie bei Verfügbarkeit eines Impfstoffes als erstes geimpft werden. Für die Risikogruppen muss also aus dem daran geknüpften Vorgehen ein Vorteil erwachsen. Weiterhin wird der Gedanke ins Spiel gebracht, dass gesellschaftliche Investitionen in dieser Richtung auch einen positiven Beitrag zu ökonomischen Überwindung der Krise leisten können, soweit man sich als Vergleich auf den Zustand eines fortgesetzten allgemeinen Shutdown mit den damit verbundenen Kosten bezieht.

Eine weitere Gruppe, für die in den letzten Wochen über ein zielgruppen-orientiertes Vorgehen diskutiert wurde, sind Kinder und Jugendliche. Im Zusammenhang mit den epidemiologischen Studien (s.o.), die eine sehr geringe Rate von Infektionen im Kindesalter, eine gute Prognose und eine geringe Infektiosität zeigen, muss hier auf die negativen Auswirkungen der unspezifischen Präventionsmaßnahmen verwiesen werden, die z.B. zu einer Verstärkung der sozialen Benachteiligung von Kindern aus bildungsfernen, sozial benachteiligten Schichten führen. Kinder stellen in diesem Sinne ebenfalls eine vulnerable Gruppe dar, denn gerade die Kinder mit geringeren Bildungschancen werden durch die Schließung von Kindergärten und Schulen besonders betroffen. Weiterhin zeigen die Studien, die z. B. zur Wirksamkeit von Schulschließungen auf den Verlauf dieser (und anderer) Epidemien vorliegen, nur eine marginale Wirkung ${ }^{23}$. Es wird daher die Empfehlung ausgesprochen, im Bereich der Kindergärten und Schulen die Rückkehr zu einer möglichst weitgehenden Normalisierung zu beschreiten.

Spezifische Präventionsmaßnahmen bedürfen definierter Instrumente der Umsetzung, daher stehen Organisationen unterschiedlicher Differenzierung im Mittelpunkt gezielter präventiver Maßnahmen. Technische und ausrüstungs- technische Systeme sind zwar notwendig, aber nicht hinreichend, damit die Institutionen (z. B. Krankenhäuser und andere Einrichtungen des Gesundheitswesens, Pflegeeinrichtungen und Betreuungseinrichtungen) mit der Bedrohung durch SARS-CoV-2/Covid-19 umzugehen lernen. Da die Bedrohung in Zeitpunkt, Ort und Art des Auftretens nicht bekannt ist und auch nicht in Erfahrung gebracht werden kann (Emergenz, s. o.), müssen die Organisationen mittel- und langfristig in ihrer Widerstandskraft gestärkt werden (Resilienz). Unter Maßgabe des engen Zeithorizontes sind folgende Ebenen von Bedeutung: Führung (Krisenkommunikation, Mitarbeiterführung in Bezug auf die Epidemie, Entlastung von Schuldzuweisung), Organisationskultur (z.B. Leitbild) und Teamkompetenz (z.B. in den Aufnahmeeinrichtungen, auf den Stationen). Zusätzlich sollten die Außenbedingungen angepasst werden: die Organisationen müssen sicher sein, dass sie nötigenfalls sofort von außen Hilfe erhalten. Kritisch für den Erfolg der Maßnahmen ist ein positives und Kompetenz-orientiertes framing, sanktionsbewehrte Maßnahmen gegen Einrichtungen sollten hintangestellt werden.

Ein ganz spezifisches Instrument, über das im Bereich der nosokomialen Infektionen zahlreiche Erfahrungen vorliegen, ist der Einsatz von regionalen Corona-Task Forces. Diese Präventionsmaßnahme, die in der Vorhaltung eines rasch verfügbaren Unterstützungsteams besteht (und weniger in einer militärisch anmutenden Eingreiftruppe - s. framing) ist besonders zur Prävention von emergenten Ereignissen und deren Folgen geeignet. Die Problematik in der Beherrschung von emergenten Ereignissen besteht generell darin, dass diese Ereignisse oft lange Zeit gar nicht auftreten. Den Mitarbeitern und den Organisationen als Ganzes kann schlicht nicht zugemutet werden, in einem monate- oder gar jahrelangen Alarmzustand zu verharren. Es reicht aus, die Organisation so wie oben beschrieben zu trainieren, und als Maßnahme für den Notfall (z. B. vorher unbekannter Corona-Fall bei Mitarbeitern oder bei Patienten/Bewohnern) zu vereinbaren, dass die Institutionen kurzfristig Hilfe und Unterstützung erhalten. So kann eine Task Force mit technischer, ablaufbezogener und personeller Unterstützung die Organisation dabei unterstützen, einen drohenden Ausbruch rechtzeitig unter Kontrolle zu bringen. Wichtig ist auch hier das positive framing, insbesondere sollten eine Sanktionierung oder andere negative Konsequenzen ausgeschlossen sein, damit keine Verzögerung bei der Anforderung entsteht.

\section{Politik und Recht bei der Bekämpfung von Pandemien}

Die Bekämpfung der Pandemie durch SARS-CoV-2/ Covid-19 wirft eine Vielzahl von Fragen auf, die von grundsätzlicher Bedeutung und für die alltägliche Praxis relevant $\operatorname{sind}^{24}$. Die juristischen Fragen betreffen das

22) Cairns/de Andrade/MacDonald, J. Health Communic. 18, 2013, 1550-1565

23) Ferguson et al. WHO Collaborating Centre for Infectious Disease Modelling, DOI: https://doi.org/10.25561/77482; Viner/ Russell/Croker/Packer/Ward/Stansfield/Mytton/Bonell/Booy, Lancet Child Adolesc. Health 2020, https://doi.org/10.1016/S23524642(20)30095-XUCL.

24) Brandneu und noch ohne Bezug zur Pandemie Kaiser, Ausnahmeverfassungsrecht, Tübingen 2020; Klafki, Neue Rechtsgrundlagen im Kampf gegen Covid-19, Verfassungsblog v. 25.3.2020, https://verfassungsblog.de/neue-rechtsgrundlagen-im-kampfgegen-covid-19/; Thielbörger/Behlert, COVID-19 und das Grundgesetz, Verfassungsblog v. 19.3.2020, https://verfassungsblog.de/ covid-19-und-das-grundgesetz/; Thielbörger/Behlert, Covid-19 und das Grundgesetz: Neue Gedanken vor dem Hintergrund neuer Gesetze, Verfassungsblog v. 30.3.2020, https://verfassungsblog.de/covid-19-und-das-grundgesetz-neue-gedankenvor-dem-hintergrund-neuer-gesetze/. 
Staatsorganisationsrecht ebenso wie die Grundrechte unserer Verfassung. Besondere Aufmerksamkeit findet dabei das am 28.3.2020 in Kraft getretene, im Eilverfahren verabschiedete und inzwischen erweiterte Gesetz zum Schutz der Bevölkerung bei einer epidemischen Lage von nationaler Tragweite ${ }^{25}$, das zu einer erheblichen Veränderung des bisher geltenden IfSG geführt hat ${ }^{26}$. Durch die Veränderungen werden einerseits die Kompetenzen des Bundes ausgeweitet, obwohl die Bundesländer das Gesetz - wie andere Bundesgesetze auch - in eigener Verantwortung ausführen. Andererseits werden die Länder ermächtigt, eigene Schutzmaßnahmen in Form von Rechtsverordnungen zu erlassen ( $\$ 32$ IfSG).

Dabei ist es von eminenter politischer und verfassungsrechtlicher Bedeutung, dass die Neufassung des Infektionsschutzgesetzes die Gewichte zwischen Legislative und Exekutive verschiebt. Diese Verschiebung stößt auf erhebliche juristische Bedenken und demokratietheoretische Zweifel ${ }^{27}$. Zwar hat der Deutsche Bundestag im Gesetzgebungsverfahren durchgesetzt, dass das Parlament selbst das Vorliegen einer epidemischen Lage von nationaler Tragweite feststellt und diese Feststellung auch wieder aufhebt. Doch nach dieser Feststellung werden dem Bundesminister für Gesundheit weitreichende Ermächtigungen zum Erlass von Rechtsverordnungen - in der Regel ohne Beteiligung des Bundesrats - und zu sofort vollziehbaren Anordnungen erteilt. Diese Ermächtigungen gestatten dem BMG Modifikationen und Ausnahmen von nahezu allen gesetzlichen und untergesetzlichen Bestimmungen des öffentlich-rechtlichen Gesundheitsrechts einschließlich der Rechtsetzung durch Selbstverwaltung ( $\$ 5$ Abs. 2 InfSG). Nach der Regelung wird das BMG ermächtigt, sogar von jeder Befugnis des IfSG und darauf gestützter Verordnungen abzuweichen, um die Abläufe im Gesundheitswesen und die Versorgung der Bevölkerung aufrecht zu erhalten. Spätestens hier halten selbst wohlwollende Rechtswissenschaftler die Grenzen des Artikel 80 Abs. 1 Satz 2 GG hinsichtlich der Bestimmung von Inhalt, Zweck und Ausmaß für überschritten ${ }^{28}$. Aber auch für alle anderen Ermächtigungsnormen gilt, dass sie mit äußerster Zurückhaltung auszulegen und anzuwenden sind, vor allem wenn und soweit sie in Grundrechte eingreifen. Diesbezüglich sind sich Rechtswissenschaftler und die in den Verfahren des einstweiligen Rechtsschutzes angerufenen Verfassungs- und Verwaltungsgerichte überwiegend einig.

Das GG kennt keine Stunde der Exekutive ${ }^{29}$. Auch vermeiden es Gerichte, Politiker und die Mehrheit der Juristen, von einem Ausnahmezustand zu sprechen ${ }^{30}$. Historisch und politisch fatal wäre die Berufung auf den umstrittenen Rechtsphilosophen Carl Schmitt (1888-1985), der in seiner Politischen Theologie postuliert hatte: „Souverän ist, wer über den Ausnahmezustand entscheidet" “31. Vielmehr mehren sich nach einigen Wochen die Stimmen, die die unter Demokratie- und Gewaltenteilungsaspekten folgenschwere Selbstentmachtung des Parlaments zugunsten einer nahezu schrankenlosen Dominanz der Exekutive sehr kritisch sehen $^{32}$. Gerade in Krisenzeiten haben die Bürgerinnen und Bürger, die demokratischen Entscheidungen Respekt und Loyalität zollen, einen Anspruch darauf, dass der Staat in allen Fragen von Legitimation und Legalität penibel alle Formen wahrt sowie Kritik demokratisch aufnimmt und nicht mit Illoyalität verwechselt. ,Der Notfall, den es zu bekämpfen gilt, bedarf der Überprüfung; die Maßnahmen, die er rechtfertigen soll, umso mehr“33. „Beispiellose Freiheitseingriffe bedürfen beispielloser Transparenz“34. Es bedarf also in schwierigen politischen Zeiten kontroverser Debatten im öffentlichen Raum, um sich gerade im Kontext von Unsicherheiten ${ }^{35}$ nicht der Illusion der Alternativlosigkeit von Zielen und Maßnahmen hinzugeben.

Weitgehende juristische Einigkeit bestand auch hinsichtlich der Bewertung von in der Krise zuerst ergriffenen Maßnahmen, mögen sich diese auch in der Form und in der konkreten Ausgestaltung von Land zu Land oder sogar mit regionalen oder örtlichen Besonderheiten unterscheiden. Parlamenten und Regierungen wurde zugutegehalten, dass sie trotz der Erfahrungen mit früheren Epidemien und der zumindest theoretisch durchgespielten Pandemieplanung von der Wucht der Erkrankung überrascht wurden. Hinzu traten unklare Informationen über Ursprung und Verbreitung des Virus in der Volksrepublik China, zögerliche Herangehensweisen in internationalen Organisationen - speziell bei der Weltgesundheitsorganisation und der Europäischen Union -, widersprüchliche Aussagen aus der Wissenschaft und dem Robert-Koch-Institut, dem nach $₫ 4$ IfSG die zentrale Rolle für die Steuerung durch Wissen zukommt, sowie eine teilweise völlig aus den Fugen geratene Berichterstattung in den Medien.

Wissenschaftler wie Gerichte billigen der Exekutive einen breiten Ermächtigungs- und Beurteilungsspielraum bei der Einschätzung der Gefahrenlage sowie bei der Auswahl und Ausgestaltung von Maßnahmen zur Eindämmung der Seuche und zur Begrenzung der Ansteckungsrisiken $\mathrm{zu}^{36}$. Dies gilt vor allem für Maßnahmen zu Beginn der Pandemie. Je länger Regelungen, insbesondere solche mit Grundrechtseinschränkungen, schon in Kraft sind, umso stärker wirkt die Verpflichtung zur kontinuierlichen Überprüfung ihrer Grundrechtsvereinbarkeit. $\mathrm{Zu}$ prüfen sind folgende Voraussetzungen: Gibt es einen verfassungslegitimen Zweck und sind die Eignung, Erforderlichkeit und Angemessenheit $=$ Verhältnismäßigkeit im engeren Sinne gegeben? Die Beschränkungen individueller Freiheiten sind mit dem erwarteten Nutzen der getroffenen Maßnahmen zur Bekämpfung der Pandemie in ein ausgewogenes Verhältnis zu bringen ${ }^{37}$. Selbst wenn eine Maßnahme von vornherein zeitlich befristet ist, besteht eine Pflicht zur re-

25) BGBl. I S. 587.

26) Rixen, NJW 2020, 1097 ff.; Klafki, Neue Rechtsgrundlagen im Kampf gegen Covid-19, Verfassungsblog v. 25.3.2020, https:// verfassungsblog.de/neue-rechtsgrundlagen-im-kampf-gegencovid-19/.

27) Bäcker, Corona in Karlsruhe, Verfassungsblog v. 25.3.2020, https://verfassungsblog.de/corona-in-karlsruhe/; Klafki, Neue Rechtsgrundlagen im Kampf gegen Covid-19, Verfassungsblog v. 25.3.2020, https://verfassungsblog.de/neue-rechtsgrundlagen-im-kampf-gegen-covid-19/.

28) Rixen, NJW 2020, 1097, $1102 \mathrm{f}$.

29) Möllers, Parlamentarische Selbstentmächtigung im Zeichen des Virus, Verfassungsblog v. 27.3.20200 (b), https:verfassungsblog. de/parlamentarische-selbstentmächtigung.im-zeichen-desvirus/; Schönberger, Die Stunde der Politik, Verfassungsblog v. 29.3.2020,httsp://verfassungsblog.de/die-stunde-der-politik.

30) So aber unter Bezugnahme auf die Diskussion um den sog. Präventionsstaat Volkmann, Der Ausnahmezustand, Verfassungsblog v. 20.3.2020, https://verfassungsblog.de/der-ausnahmezustand/; und differenzierend Mehring, Carl Schmitt und die Pandemie, Teile I und Ii, VerfBlog v. 11.5 und 12.5.2020, https://verfassungsblo.de/carl-schmitt-und-die-pandemie-teil-i bzw. teil-ii/.

31) Schmitt, Politische Theologie - Vier Kapitel zur Lehre von der Souveränität, 1922, S. 9

32) Exemplarisch Kingreen, Whatever it takes? Der demokratische Rechtsstaat in Zeiten von Corona, Verfassungsblog v. 20.3.2020, http://verfassungsblog.de/whatever-it-takes/.

33) Möllers, Über den Schutz der Parlamente vor sich selbst in der Krise, Verfassungsblog v. 20.3.2020, https://verfassungsblog.de/ über-den-schutz-der-parlamente-vor-sich-selbst-in-der-krise/.

34) Spamann, Beispiellose Freiheitseingriffe brauchen beispiellose Transparenz, Verfassungsblog v. 4.4.2020, http://verfassungsblog.de/beispiellose-freiheitseingriffe-brauchen-beispiellosetransparenz/.

35) Frankenberg, COVID-19 und der juristische Umgang mit Unsicherheit, Verfassungsblog v. 25.4.2020, https://verfassungsblog. de/covid-19-und-der-juristische-umgang-mit-unsicherheit/.

36) Exemplarisch VGH München, NJW 2020, 1236, 1240; OVG Bautzen, NJW 2020, $1384 \mathrm{ff}$.

37) BVerfG, NJW 2020, 1429 
gelmäßigen Evaluierung, insbesondere im Hinblick auf die Erforderlichkeit und die Angemessenheit. Sollte sich die Unverhältnismäßigkeit einzelner Regelungen herausstellen, sind diese isoliert unverzüglich auch vor Ablauf des Geltungszeitraums eines Gesamtpakets aufzuheben ${ }^{38}$.

Schließlich besteht auch Einigkeit, dass viele der von Bund und Ländern erlassenen Rechtsakte erhebliche Eingriffe in wesentliche Freiheitsrechte der Menschen in Deutschland bedeuten. In diese Grundrechte darf durch Gesetz oder auf Grund eines Gesetzes eingegriffen werden. Generell werden nur wenig Zweifel geäußert, dass die Regelungen des Infektionsschutzgesetzes, insbesondere die $\int \$ 28$ Abs. 1 und 32 des IfSG als ausreichende Rechtsgrundlagen für Verordnungen, Allgemeinverfügungen und Einzelverfügungen angesehen werden ${ }^{39}$.

Ganz wesentlich kommt es für die Beurteilung der Rechtmäßigkeit auf die Verhältnismäßigkeit der Eingriffe an. Diese Prüfung darf nicht mit der „Holzhammermethode“"40 erfolgen. Jeder Eingriff muss ein legitimes Ziel in geeigneter, erforderlicher und angemessener Weise verfolgen. Kohärenz ist notwendige ,Begleitmusik zum infektionsschutzrechtlichen Tanz"“41. Unzweifelhaft sind der Schutz der Bevölkerung und die Verhinderung der Überlastung des Gesundheitswesens legitime Ziele für Grundrechtseingriffe. Auch dürften Maßnahmen zur Verringerung oder gar Verhinderung von sozialen Kontakten grundsätzlich geeignet sein, die Infektionsgeschwindigkeit des Virus zu verlangsamen oder die Pandemie zum Stillstand zu bringen. Bei der Auswahl der Maßnahmen besteht - zumindest zu Beginn einer Pandemie - ein weiter Beurteilungsspielraum. Dabei gilt der allgemeine Grundsatz der Gefahrenabwehr, dass an die Wahrscheinlichkeit des Schadenseintritts umso geringere Anforderungen zu stellen sind, je größer und folgenschwerer die Schäden sein können. Inwieweit Ausweispflichten, die Untersagung der Nutzung von Zweitwohnungen oder das Verbot touristischer Reisen geeignet sind, legitime Ziele des Infektionsschutzes zu erreichen, darf mehr als bezweifelt werden $^{42}$. Das gilt erst recht für das Verbot, die Grenzen eines Bundeslands zu überschreiten, oder gar das Gebot, ein Bundesland zu verlassen. Die Landesregierung von Mecklenburg-Vorpommern hat als bedeutendes Urlaubsland nicht nur der Tourismusbranche im Land schweren Schaden zugefügt, sondern auch einen Tiefpunkt des föderalen Egoismus markiert. Die Ab- und Ausweisung an Ländergrenzen sollte im Jahr 30 der Wiedervereinigung politisch und juristisch tabu sein. Das gilt besonders für den Fall, dass Bürger aus weniger betroffenen Landesteilen in Landesteile mit höherer Krankheitslast ausgewiesen werden, nur weil sie dort mit ihrem Erstwohnsitz gemeldet sind. Die Landesregierung von Schleswig-Holstein war cleverer und hat die Anreise zu einem Zweitwohnungssitz unabhängig von der Lage ihres Erstwohnsitzes untersagt. Das zuständige OVG hat in einer Güterabwägung zugunsten des öffentlichen Interesses an einer Eindämmung der Pandemie entschieden ${ }^{43}$. Dagegen haben das OVG Berlin-Brandenburg ${ }^{44}$ wie schon die Vorinstanz ${ }^{45}$ Zweifel an der Geeignetheit und der Verhältnismäßigkeit einer entsprechenden Reisebeschränkung durch Allgemeinverfügung eines Landkreises artikuliert und deshalb diese Verfügung aufgehoben.

Trefflich streiten lässt sich darüber, ob die vielen Einschränkungen in allen Einzelfällen erforderlich oder ob nicht andere weniger belastende Maßnahmen verfügbar sind (Lepsius ${ }^{46}$ beklagt in diesem Zusammenhang einen Niedergang grundrechtlicher Denkkategorien). In diesem Zusammenhang ist zu prüfen, wann spezifische Präventionsmaßnahmen für besonders gefährdete Risikogruppen allgemeine Gebote und Verbote abmildern oder ersetzen können ${ }^{47}$. Vor allem aber ist mit zunehmender Dauer der Freiheitsbeschränkungen immer wieder zu prüfen, ob diese nicht außer Verhältnis zum verfolgten Zweck stehen ${ }^{48}$. Bei der Prüfung reicht es nicht aus, allein auf die Erkrankungs- und Todeszahlen abzustellen. Das Grundrecht auf Leben und körperliche Unversehrtheit begründet sicher besondere Schutzpflichten des Staates, ist aber nicht per se anderen Grundrechten übergeordnet. Vor allem ist es nicht zulässig, Leben gegen Leben aufzurechnen und abzuwägen. Die diesbezüglichen Ausführungen des BVerfG zur Nichtigkeit einer Abschussermächtigung im Luftsicherheitsgeset $\mathrm{z}^{49}$ sind Pflichtlektüre für alle, die (verfassungs-)rechtliche Überlegungen zur Corona-Pandemie anstellen. Ansonsten wäre eine Debatte um ein Tempolimit auf Autobahnen schlicht überflüssig ${ }^{50}$. Daher wird kontinuierlich zu prüfen sein, inwieweit Ausnahmeregelungen von strikten Regulierungen ausgeweitet und die Zielsetzung der Regelungen durch Auflagen mit milderen Vorgaben (Abstandsgebot, Maskenpflicht ...) erreicht werden kann. Daraus lässt sich aber keine allgemeine Regel für alle denkbaren Fallgestaltungen ableiten, sondern „,nur“ die allgemeine Verpflichtung, für jede Fallkonstellation eine Abwägung konkurrierender Grundrechte im Kontext des jeweiligen Erkenntniszustands vorzunehmen. Was gestern noch unausweichlich war, kann schon morgen fehlerhaft sein. Diese Anforderungen gelten im Übrigen nicht nur für bereits beschlossene Maßnahmen, sondern erst Recht für das, was in Erwägung gezogen wird. Das betrifft etwa die diskutierte Ausstellung von lmmunitätsausweisen oder den Einsatz von Tracing- und TrackingApps, deren Zulässigkeit von der technischen Ausgestaltung (zentrale oder dezentrale Speicherung der Daten) und der Freiwilligkeit der Anwendung abhängig sein wird.

Die verfassungsrechtlichen Problemstellungen sind auch relevant bei der Beurteilung von Maßnahmen zur Lockerung der Beschränkungen. Diese müssen insbesondere dem Prüfmaßstab des allgemeinen Gleichheitssatzes des Art. 3 Abs. 1 GG entsprechen. Danach darf weder wesentlich Gleiches willkürlich ungleich noch wesentlich Ungleiches willkürlich gleichbehandelt werden ${ }^{51}$. Das Gericht differenziert dabei zwischen Ungleichbehandlungen geringerer und größerer Intensität. Die bisher verfügten Einschränkungen und deren Ausnahmen bieten eine Fülle von Vergleichsmöglichkeiten ${ }^{52}$. Diese beschäftigen bereits die

38) Katzenmeier, MedR 2020, $461 \mathrm{ff}$.

39) BVerfG, NJW 2020: 1429; Wissenschaftlicher Dienst des Deutschen Bundestags, Kontaktbeschränkungen zwecks Infektionsschutz: Grundrechte, WD 3 - 3000 - 079/20, Berlin 2020, S. 15.

40) Hofmann, Verhältnismäßigkeit mit der Holzhammermethode, Verfassungsblog v. 13.4.2020, https://verfassungsblog.de/verhaeltnismaessigkeit-mit-der-holzhammermethode/.

41) Michl, Die Kohärenz als Begleitmusik zum infektionsschutzrechtlichen Tanz, Verfassungsblog v. 28.4. https://verfassungsblog.de/die-kohaerenz-als-begleitmusik-zum-infektionsschutzrechtlichen-tanz/.

42) Wissenschaftlicher Dienst des Deutschen Bundestags, Kontaktbeschränkungen zwecks Infektionsschutz: Grundrechte, WD 3 $-3000-079 / 20$, Berlin 2020, 2020, S. 16f.m. N. aus der Rechtsprechung.

43) OVG Schleswig, NJW 2020, $1382 \mathrm{ff}$.

44) OVG Berlin-Brandenburg, NJW 2020, $1454 \mathrm{ff}$

45) VG Potsdam, COVuR 1/2020, $64 \mathrm{f}$. und $65 \mathrm{ff}$.

46) Lepsius, Vom Niedergang grundrechtlicher Denkkategorien in der Corona-Pandemie, Verfassungsblog v. 6.4.2020, https:// verfassungsblog.de/vom-niedergang-grundrechtlicher-denkkategorien-in-der-corona-epidemie/.

47) Huster, Grenzen der Solidarität, Verfassungsblog v. 22.3.2020, https://verfassungsblog.de/grenzen-der-solidaritaet/.

48) Berliner VerfGH, NJW 2020, $1505 \mathrm{ff}$

49) BVerfGE 115, $118 \mathrm{ff}$.

50) Kingreen, Whatever ist takes? Der demokratische Rechtsstaat in Zeiten von Corona, Verfassungsblog v. 20.3.2020, http://verfassungsblog.de/whatever-it-takes/.

51) St. Rspr. d. BVerfG seit BVerfGE 1, 14, 16; 4, 144, 155.

52) Beispiele beim Wissenschaftlichen Dienst des Deutschen Bundestags, Kontaktbeschränkungen zwecks Infektionsschutz: Grundrechte, WD 3 - 3000 - 079/20, 2020, S. $31 \mathrm{ff}$. 
Rechtsprechung, gegebenenfalls wie bei der Öffnung von Ladenlokalen mit einer Verkaufsfläche bis zu 800 Quadratmetern mit unterschiedlichem Ausgang. So ist es folgerichtig, dass das BVerfG in jüngster Zeit das ausnahmslose Verbot von Gottesdiensten mit Gläubigen vor Ort gekippt hat - übrigens auf Antrag einer muslimischen Gemeinde ${ }^{53}$ - nachdem es noch einige Wochen zuvor Anträge christlicher Gläubiger auf Durchführung von Ostergottesdiensten abgelehnt hatte ${ }^{54}$. Auch das ausnahmslose Demonstrationsverbot wurde im Laufe der Zeit durch das höchste Gericht gelockert ${ }^{55}$. Zudem sehen Landesverfassungsgerichte jetzt genauer hin als zu Beginn der Pandemie ${ }^{56}$ und treten obrigkeitsstaatlichen Tendenzen in der Krise entgegen ${ }^{57}$.

Liegt eine Ungleichbehandlung innerhalb einer Vergleichsgruppe vor, kann diese gleichwohl gerechtfertigt sein, wenn das Differenzierungsziel legitim ist, die Differenzierungskriterien zur Erreichung des Ziels geeignet sind und die Differenzierung erforderlich und angemessen ist. Diese Prüfung steht vor allem dann an, wenn die Politik spezielle Maßnahmen für besondere Risikogruppen verhängen sollte. Insbesondere das Kriterium Alter dürfte verfassungsrechtlich sehr unterschiedlich bewertet werden, selbst wenn das Grundgesetz, anders als diverse internationale Konventionen, das Alter nicht als besonderen Schutztatbestand vor Diskriminierung benennt. Ausdrücklich genannt ist in Art. 3 Abs. 3 GG der Tatbestand der Behinderung, dem bisher nur begrenzte Aufmerksamkeit gewidmet wird.

Aus dem Schutz der Menschenwürde, dem Grundprinzip der Verfassung nach Art. 1 Abs. 1 S. 1 GG, leitet das BVerfG die Voraussetzung ab, dass der Mensch in seiner fragilen und individuellen und sozialen Existenz über sich nach eigenen Maßstäben verfügen kann und nicht in Lebensformen gedrängt werden darf, die in einem unauflösbaren Widerspruch zum eigenen Selbstbild und Selbstverständnis stehen ${ }^{58}$. Der Mensch darf dabei nicht zu einem Objekt staatlichen Handels herabgewürdigt werden. Einige der ergriffenen Maßnahmen, speziell deren noch weitgehend unerforschte gesundheitliche und soziale Wirkungen, bergen zumindest ein solches Potenzial ${ }^{59}$. Auf der anderen Seite leitet das Gericht aus der Menschenwürde in Verbindung mit dem Grundrecht auf Leben und körperliche Unversehrtheit eine staatliche Schutzpflicht für das schwache Leben $\mathrm{ab}^{60}$. Konkret sind also die freie und gleiche Persönlichkeitsentfaltung in Würde mit der Würde der besonders gefährdeten Risikogruppen abzuwägen ${ }^{61}$. Daraus ergibt sich schon sachlogisch, dass es gerade in diesen Konstellationen keine rechtmäßigen „Pauschalmaßnahmen“ geben kann. Ein allgemeines Sonderopfer bestimmter Gruppen für die Volksgesundheit kommt nicht oder nur in sehr engen Grenzen in Betracht ${ }^{62}$. Erst recht kann ein Mensch mit Vorerkrankungen nicht verlangen, dass eine Anlaufpraxis zum Test auf das Coronavirus in der Nachbarschaft geschlossen wird ${ }^{63}$.

Mit zunehmender Dauer von Zuständigkeitsverlagerungen und Grundrechtseinschränkungen steigt der Druck, die Maßnahmen nicht nur auf eine zuverlässige epidemiologische Grundlage zu stellen, sondern auch zu transparenten und nachvollziehbaren Abwägungsprozessen zurückzukehren, die rechtsstaatlichen Maßstäben genügen. Das BVerfG hat in fast 70 Jahren seiner Geschichte klare Entscheidungen getroffen ${ }^{64}$ und die Rechtswissenschaft hat vielfältige Orientierungshilfen gegeben ${ }^{65}$. Not kennt also durchaus Gebot ${ }^{66}$. Der demokratische und soziale ,Staat der Mitte" ${ }^{67}$ hat sich - anders als in illiberalen und autoritären Systemen - bisher in Krisen bewährt. Er sollte es auch weiterhin tun.

\section{Fazit}

Zur epidemiologischen Beschreibung der Epidemie sind zuverlässige Häufigkeitsmaße genauso wichtig wie sinnvolle Teststrategien, die eine zu hohe Rate falsch-positiver Befunde vermeiden. Schwerpunkte sind die Infektionen im hohen Alter bei Vorliegen von Begleiterkrankungen sowie die nosokomiale bzw. herdförmige Ausbreitung; aus bislang ungeklärten Gründen spielen Kinder in der Dynamik der Epidemie eine untergeordnete Rolle. Die Präventionsmaßnahmen können sich nicht auf allgemeine Maßnahmen (z.B. social distancing) oder Nachverfolgung beschränken, sondern sollten in Analogie $\mathrm{zu}$ anderen Epidemien den spezifischen Schutz von Risikogruppen mit einschließen. Die deutsche Verfassung kennt für den Fall einer Pandemie keinen Ausnahmezustand, der eine Abweichung von Aufgabenzuordnungen und Kompetenzen des föderalen Staatsaufbaus und der demokratischen Gewaltenteilung erlauben würde. Zwar kann in Grundrechte auch der gesamten Bevölkerung eingegriffen werden, doch bedürfen Eingriffe stets einer legitimen Rechtfertigung und eines transparenten Abwägungsprozesses zwischen konkurrierenden Grundrechten sowie zwischen Grundrechten und Schutzpflichten des Staates. Je länger Beschränkungen andauern, desto stärker ist der Zwang zu kontinuierlicher Evaluation ausgeprägt, speziell in Bezug auf die Beachtung der Verhältnismäßigkeit.

53) Pressemitteilung Nr. 28a/2020 v. 29.4.2020.

54) BVerfG, NJW 2020, $1427 \mathrm{ff}$.

55) BVerfG, NJW 2020, 1426f.; NJW 2020, 1505.

56) Heumann/Holzgang, Die Stunde der Verfassungsgerichte, Verfassungsblog v. 30.4.2020, https://verfassungsblog.de/die-stundeder-verfassungsgerichte/.

57) Kumm, Gegen obrigkeitsstaatliche Tendenzen in der Krise, Verfassungsblog v. 20.4.2020, https://verfassungsblog.de/gegenobrigkeitsstaatliche-tendenzen-in der-krise/.

58) BVerfG, NJW 2020, $905 \mathrm{ff}$.

59) Wissenschaftlicher Dienst des Deutschen Bundestags, Kontaktbeschränkungen zwecks Infektionsschutz: Grundrechte, WD 3 $-3000-079 / 20$, Berlin 2020, S. $37 \mathrm{f}$

60) BVerfGE 88, 203, $251 \mathrm{f}$.

61) Kube, Leben in Würde - Würde des Lebens, Verfassungsblog v. 2.4.2020, http://verfassungsblog/leben-in-wuerde-wuerdedes-lebens/ und wohl auch Huster: Grenzen der Solidarität, Verfassungsblog v. 22.3.2020,https://verfassungsblog.de/grenzen-der-solidaritaet/.

62) Albers, Sonderopfer für die Volksgesundheit: Freiheitsbeschränkungen zum Lebensschutz in der Coronakrise, VerfBlog v. 11.5.2020, https://verfassungsblog.de/sonderopfer-fuer-die-volksgesundheit/.

63) VG Bremen, COVuR 1/2020, $53 \mathrm{ff}$.

64) Lamprecht, Ich gehe bis nach Karlsruhe - Eine Geschichte des BVerfG, 2009.

65) Heinig/Schorkopf (Hrsg.), 70 Jahre Grundgesetz - In welcher Verfassung ist die Bundesrepublik?, Göttingen 2019; Lepsius, Vom Niedergang grundrechtlicher Denkkategorien in der CoronaPandemie, Verfassungsblog v. 6. 4.2020, https://verfassungsblog. de/vom-niedergang-grundrechtlicher-denkkategorien-in-dercorona-epidemie/.

66) Kaiser, Ausnahmeverfassungsrecht, 2020, S. $207 \mathrm{ff}$.

67) Ipsen, Der Staat der Mitte - Verfassungsgeschichte der Bundesrepublik Deutschland, 2009. 